\title{
Somatic Chromosomes of Acridotheres fuscus fuscus Wagler and Acridotheres tristis tristis Linnaeus
}

\author{
G. P. Sharma, O. P. Mittal and N. Gupta \\ Department of Zoology, Panjab University, Chandigarh, India
}

Received October 4, 1977

The birds occupy a unique position amongst the vertebrates because of their high number of chromosomes. However, it is perhaps the unmanageable analysis of their microchromosomes which accounts for the class Aves being little known cytologically. Some of the other reasons for their being so, are the difficulties involved during their capture and breeding. Nevertheless, with the advent of the improved cytological techniques, the avian karyological studies have progressed to some extent recently. A few such studies have been made even on the passerine birds (see Makino 1951, Udagawa 1952, Makino and Baldwin 1954, Ray-Chaudhuri 1973, Mittal and Kanwaljit 1975, Sharma et al. 1976).

One of the major achievements with regard to the cytogenetics of birds during the recent years has been the identification of a W-chromosome in the somatic cells of the female of the various species, thus confirming the sex mechanism of the $\mathrm{ZZ}$ / ZW type.

The present studies on two passerine birds namely, Acridotheres fuscus fuscus and $A$. tristis tristis have been undertaken with a view to add some more information to the existing knowledge about the avian karyology. An attempt has also been made to find out the cytological relationships between them and their allied forms, if any.

\section{Material and methods}

The males and females of two species of myna, Acridotheres fuscus fuscus (Jungle myna) and $A$. tristis tristis (common myna), belonging to the family Sturnidae, order Passeriformes, constitute the material for the present studies. The birds were injected intraperitoneally with $1 \mathrm{ml}$ of $0.1 \%$ aqueous colchicine solution for at least 3 hours prior to their sacrifice. The chromosome preparations were made from the bone-marrow cells of both the sexes using the flame-drying technique. The slides were stained in carbol-fuchsin, dehydrated in n-butyl alcohol and mounted in Euparal.

\section{Observations}

The diploid number of 74 and 80 chromosomes for Acridotheres fuscus fuscus and $A$. tristis tristis has been determined from 35 and 40 metaphase cells respectively. This diploid number in A. fuscus fuscus comprises 12 macro- and 62 microchromosomes (Figs. 1-4), while in A. tristis tristis there are 12 macro- and 68 microchromo- 
somes (Figs. 5-8). Amongst the microchromosomes there are a few elements which fall in between the macro- and micro-chromosomes according to their size and they have been named as the "intermediate or medium-sized ones". The number of macrochromosomes is constant in all the plates studied, while that of the micro-

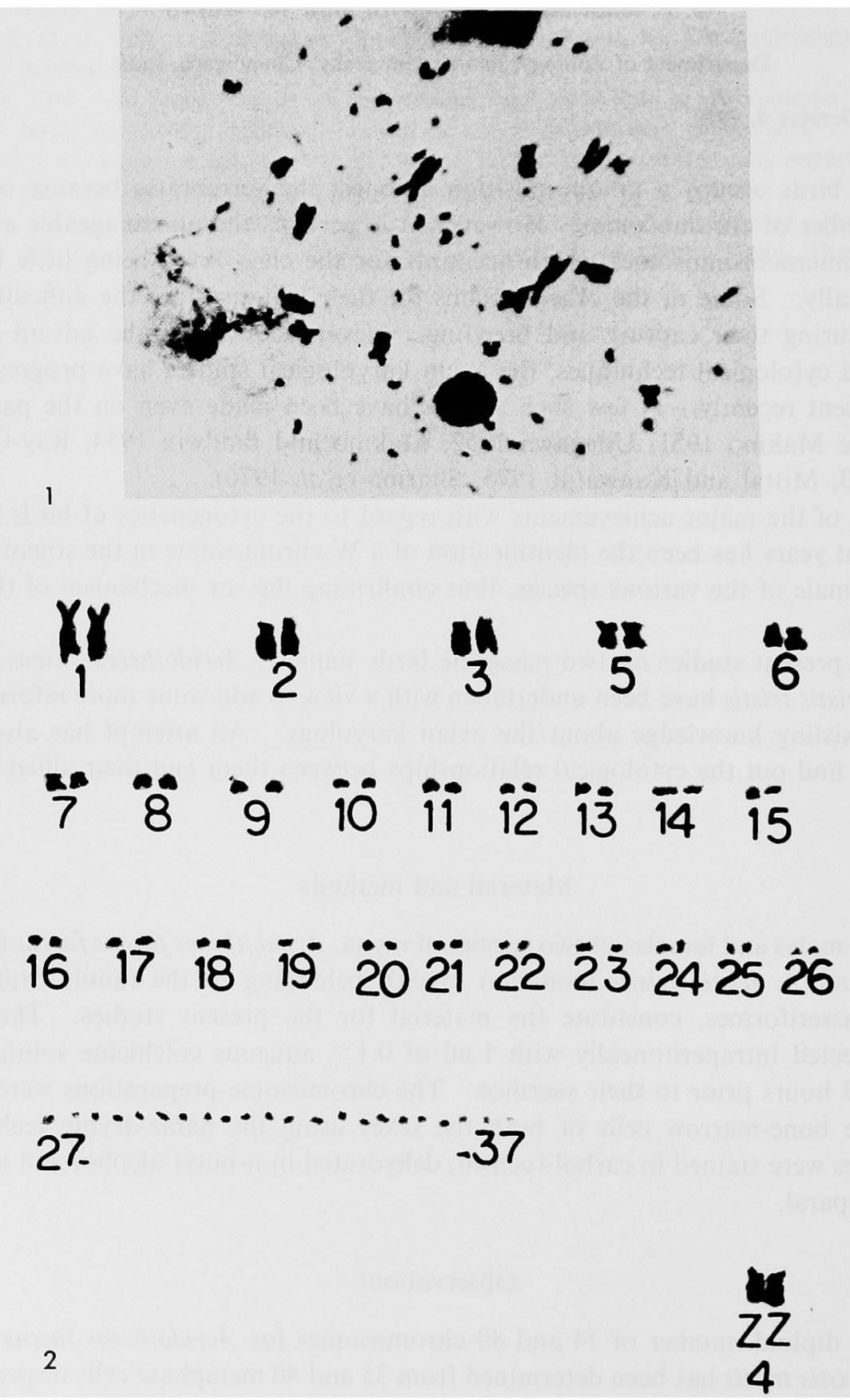

Figs. 1-2. 1, metaphase of Acridotheres fuscus fuscus (Male). $\quad \times 4500.2$, karyotype prepared from Fig. 1. 
chromosomes is slightly variable. The intermediate elements are more prominent in $A$. fuscus fuscus than in A. tristis tristis. In the former species, 4 pairs of intermediate elements have been identified. The 7 th pair of chromosomes, which is an intermediate, has a clear morphology in some cells,. However, in A. tristis

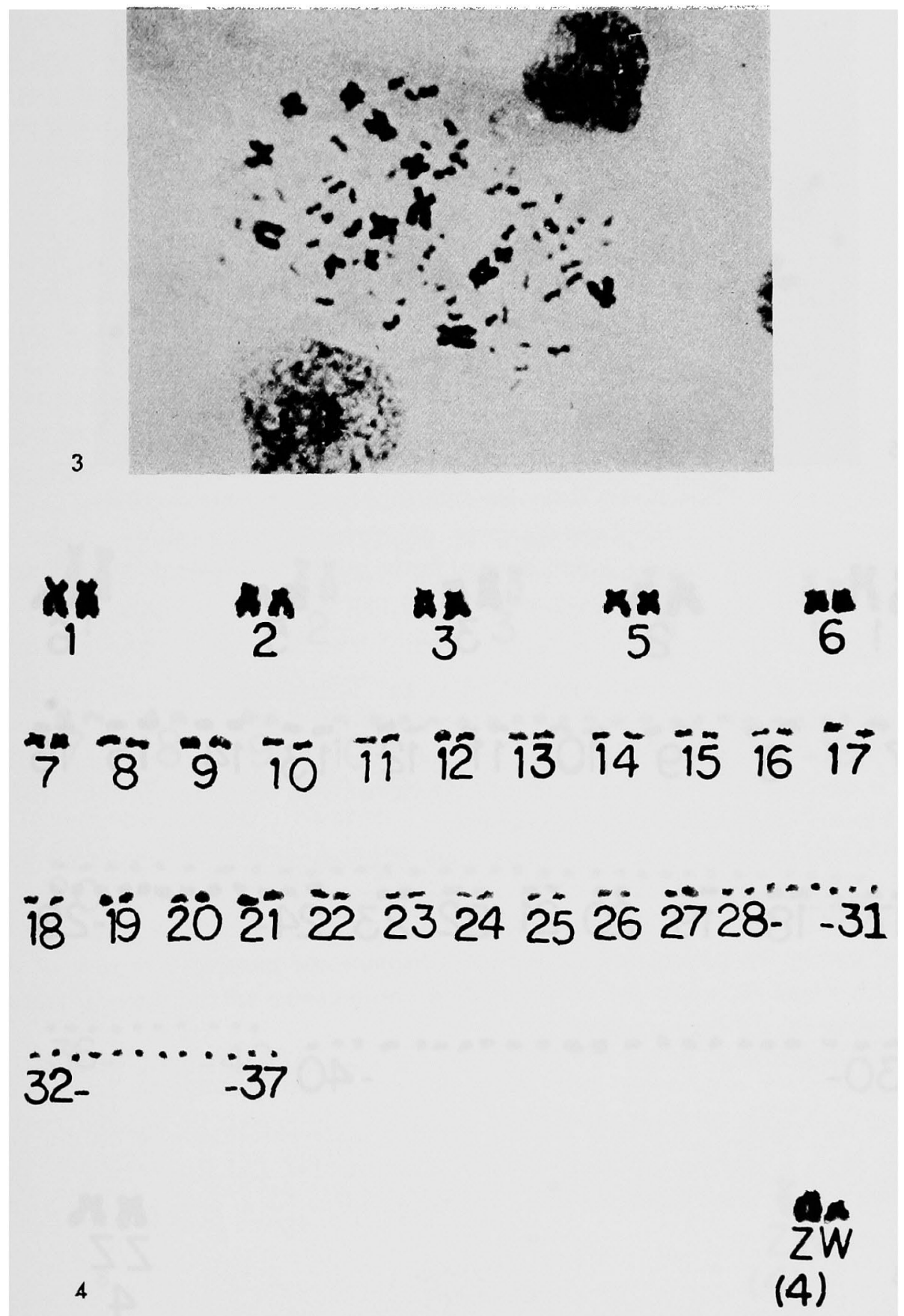

Figs. 3-4. 3, metaphase of Acridotheres fuscus fuscus (Female). $\times 4500.4$, karyotype prepared from Fig. 3. 
tristis only1-2 pairs of these elements have been recognized.

Morphological analysis

Due to small size of the microchromosomes, their centromeric positions are

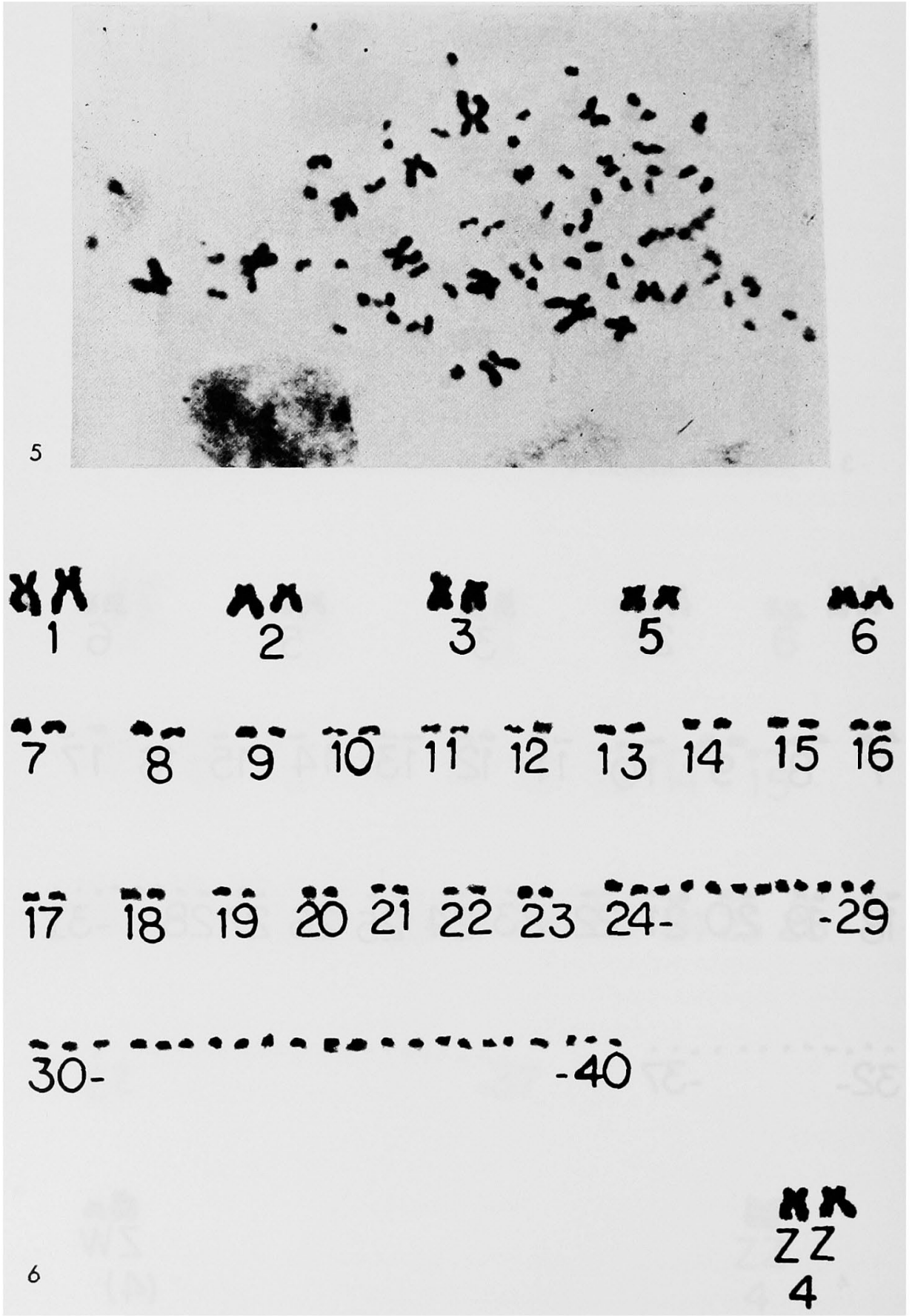

Figs. 5-6. 5, metaphase of Acridotheres tristis tristis (Male). $\times 4500.6$, karyotype prepared from Fig. 5. 
not clear and hence their morphology cannot be precisely determined. However, the macro- and some of the intermediate chromosomes have been classified into different groups on the basis of their length and centromeric positions. The system

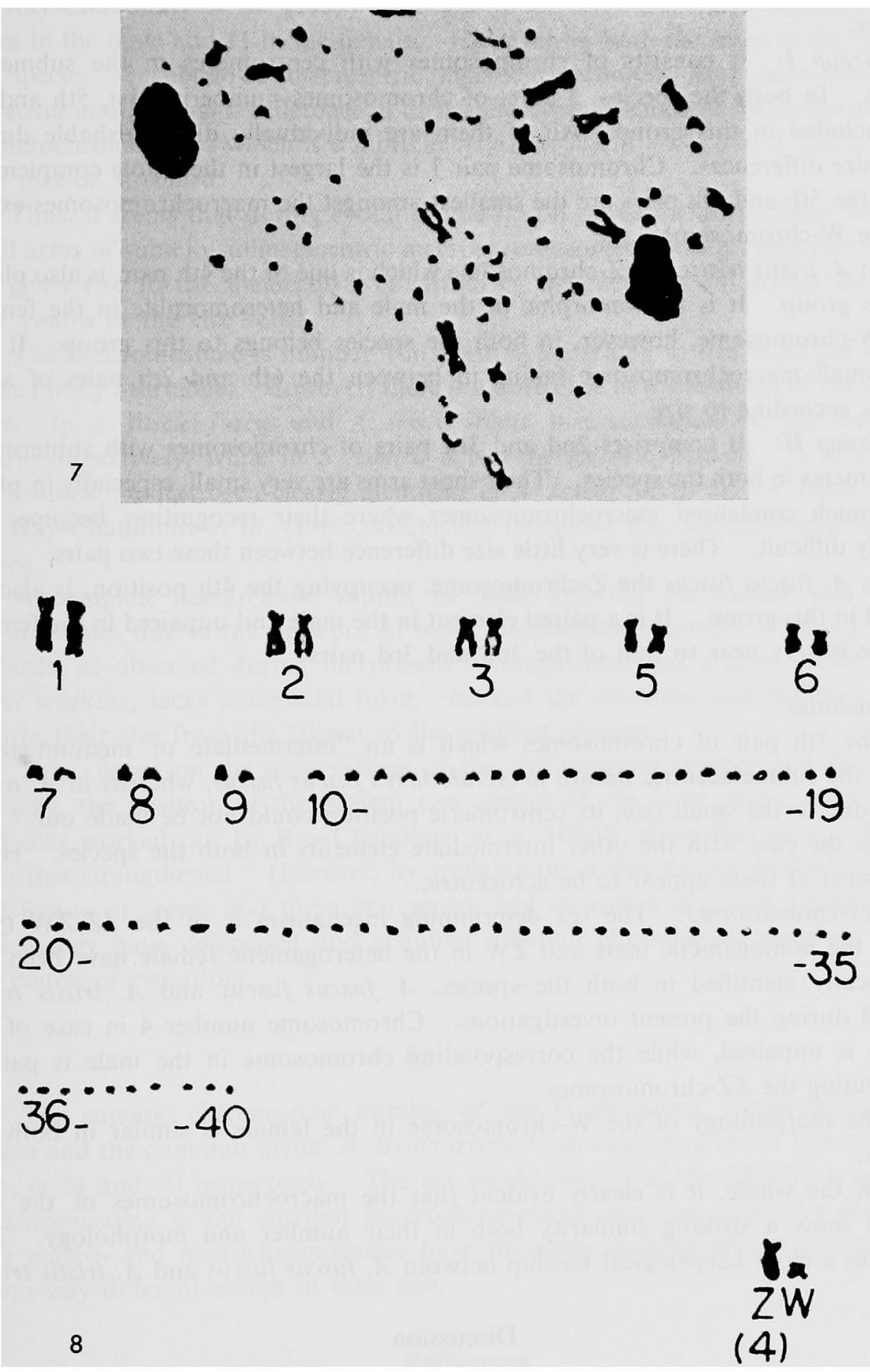

Figs. 7-8. 7, metaphase of Acridotheres tristis tristis (Female). $\times 4500 . \quad 8$, karyotype prepared from Fig. 7. 
of nomenclature of the chromosomes which is followed here is the same as proposed by Levan et al. (1964). Pairing of the microchromosomes has also been attempted to some extent (Figs. 2, 4, 6 and 8).

The macrochromosomes in the two species are divided into the following two groups:

Group I: It consists of chromosomes with centromeres in the submedian region. In both the species, 3 pairs of chromosomes numbering 1st, 5th and 6th are included in this group. All of them are individually distinguishable due to their size differences. Chromosome pair 1 is the largest in the whole complement, while the 5th and 6th pairs are the smallest amongst the macrochromosomes except for the W-chromosomes.

In $A$. tristis tristis, the Z-chromosome which is one of the 4th pair, is also placed in this group. It is monomorphic in the male and heteromorphic in the female. The W-chromosome, however, in both the species belongs to this group. It is a very small macrochromosome falling in between the 6th and 7th pairs of autosomes, according to size.

Group II: It comprises 2 nd and $3 \mathrm{rd}$ pairs of chromosomes with subterminal centromeres in both the species. Their short arms are very small, especially in plates with much condensed macrochromosomes where their recognition becomes extremely difficult. There is very little size difference between these two pairs.

In $A$. fuscus fuscus the Z-chromosome, occupying the 4th position, is also included in this group. It is a paired element in the male and unpaired in the female. Its size is very near to that of the 2 nd and 3 rd pairs.

\section{Intermediates}

The 7th pair of chromosomes which is an "intermediate or medium-sized" shows the submetacentric nature in Acridotheres fuscus fuscus, whereas in A. tristis tristis due to the small size, its centromeric position could not be made out. The same is the case with the other intermediate elements in both the species. However, most of them appear to be acrocentric.

Sex-chromosomes: The sex determining mechanism is of the $\mathrm{ZZ} / \mathrm{ZW}$ type. $\mathrm{ZZ}$ in the homogametic male and $\mathrm{ZW}$ in the heterogametic female have been unequivocally identified in both the species, A. fuscus fuscus and A. tristis tristis studied during the present investigations. Chromosome number 4 in case of the female is unpaired, while the corresponding chromosome in the male is paired, constituting the ZZ-chromosomes.

The morphology of the W-chromosome in the female is similar in both the species.

On the whole, it is clearly evident that the macrochromosomes of the two species show a striking similarity both in their number and morphology. This indicates a close karyological kinship between $A$. fuscus fuscus and A. tristis tristis.

\section{Discussion}

The present discussion is mainly based on a comparison of the karyotypes of Acridotheres fuscus fuscus and A. tristis tristis with that of Sturnus contra, belonging 
to the same family Sturnidae. Ray-Chaudhuri et al. (1969) observed 68 chromosomes in Sturnus contra, whereas during the present investigation on $A$. fuscus fuscus and $A$. tristis tristis, 74 and 80 chromosomes have been recorded, respectively.

Ray-Chaudhuri et al. (1969) have shown 12 submetacentric macrochromosomes in the male and 11 in the female. However, in both the sexes of the present two species 12 subtelo-submetacentric macrochromosomes have been observed. Moreover another major difference in their karyotypes is concerned with the number of microchromosomes which is 6 more in A. fuscus fuscus and 12 in A. tristis tristis than that of $S$. contra.

Thus, it seems that during evolution the fusion of microchromosomes with the small arms of subtelo-submetacentric macrochromosomes of the present two species has given rise to the metacentric condition of the macrochromosomes met with in $S$. contra or the vice versa.

The Z-chromosome is number 4 in position according to size in all the species of the family Sturnidae. However, there is a difference in the position of the centromere. In $A$. fuscus fuscus and $A$. tristis tristis, it is subtelocentric and submetacentric respectively, while in $S$. contra it is a metacentric element. A submedian W-chromosome has been clearly identified in A. fuscus fuscus and A. tristis tristis, but Ray-Chaudhuri et al. (1969), could not recognize this sex-chromosome in $S$. contra.

A complete demarcation cannot be made between the macro- and microchromosomes due to the presence of some intermediate elements. The karyotype of birds, as observed during the present investigations, and also recorded by the other workers, lacks a bimodal form. Instead the chromosomes depict a gradation in their size from the largest to the smallest element.

Thus, based on the observations of the chromosome number and their morphology, the position of the present two species of the same genus, Acridotheres and that worked out by Ray-Chaudhuri et al. (1969) of another genus, Sturnus, is further strengthened. However, by applying the Robertsonian method of fusion and fission, it seems that these two genera had at least a common ancestor from which they have descended and acquired different chromosome numbers during the course of evolution.

\section{Summary}

The somatic chromosome number of the jungle myna, Acridotheres fuscus fuscus and the common myna, A. tristis tristis, both belonging to the family Sturnidae, is 74 and 80 respectively. The sex mechanism is of the $\mathrm{ZZ} / \mathrm{ZW}$ type. The W-chromosome is the smallest macrochromosome and can easily be identified. The macro- and microchromosomes have the same staining affinity and they are in no way different except in their size.

\section{References}

Levan, A., Fredga, K. and Sandberg, A. A. 1964. Hereditas (Lon.) 52: 201-220.

Makino, S. 1951. Atlas of the Chromosome Numbers in Animals (Second edition), The Iowa 
State College Press, Ames, Iowa.

— and Baldwin, P. H. 1954. Cytologia 19: 217-224.

Mittal, O. P. and Kanwaljit, 1975. Second All India Congress of Cytology and Genetics, Udaipur, Abstracts: 74.

Ray-Chaudhuri, S. P., Sharma, T. and Ray-Chaudhuri, R. 1969. Chromosoma 26: 148-168. Ray-Chaudhuri, R. 1973. In: Cytotaxonomy and Vertebrate Evolution. Edt. Chiarelli and Capanna. United States Edition, Academic Press Inc. (London) Ltd.

Sharma, G. P., Mittal, O. P. and Gupta, Nupur. 1976. L. C. Dunn and Th. Dobzhansky memorial symposium on genetics Manasa Gangotri, Mysore, Programme and Abstracts: 30-31. Udagawa, T. 1952. Cytologia 17: 311-316. 